\title{
Guide to Receptors and Channels, 2nd edition (2007 Revision)
}

The great proliferation of drug targets in recent years has driven the need to organise and condense the information in a logical way. This is the underlying reason for this Guide to Receptors and Channels, distributed with the British Journal of Pharmacology. Our intent is to produce an authoritative but user-friendly publication, which allows a rapid overview of the key properties of a wide range of established or potential pharmacological targets. The aim is to provide information succinctly, so that a newcomer to a particular target group can identify the main elements 'at a glance'. It is not our goal to produce all-inclusive reviews of the targets presented; references to these are included in the Further Reading sections of the entries. The Guide to Receptors and Channels presents each entry, typically a circumscribed target class family on, wherever possible, a single page, so as to allow easy access and rapid oversight.

Targets have been selected for inclusion where there is sufficient pharmacological information to allow clear definition or where, in our view, there is clear interest in this molecular class from the pharmacological community. Our philosophy has been to present data on human receptors wherever possible, both in terms of structural information and pharmacology. To this end, the Ensembl ID allows rapid access through a free online database (http://www.ensembl.org/) to several other species, including mouse and rat. From this database, links are also provided to structural information in a number of formats. Where structural or pharmacological information is not available for human targets, we have used data from other species. A priority in constructing these tables was to present agents which represent the most selective and which are available by donation or from commercial sources, now or in the near future.

The Guide is divided into seven sections, which comprise pharmacological targets of similar structure/function. These are 7TM receptors, transmitter-gated channels, ion channels, catalytic receptors, nuclear receptors, transporters and enzymes. In comparison with the First Edition of the Guide to Receptors and Channels (Alexander et al., 2004), we have added an additional group of records, expanding the total by approximately $25 \%$. Although for many of these records, a full pharmacological comparison is unavailable, there is sufficient interest from pharmacologists to warrant their inclusion.

The Editors of the Guide have compiled the individual records, taking advice from many Consultants (listed on page S2). With each record, an indication is given of the status of the nomenclature, as proposed by Nomenclature Committees of the International Pharmacological Congress (NC-IUPHAR), published in Pharmacological Reviews. Where this guidance is lacking, advice from several prominent, independent experts has been obtained to produce an authoritative consensus, which attempts to fit in within the general guidelines from NC-IUPHAR (Vanhoutte et al., 1996). Tabulated data provide ready comparison of selective agents and probes (radioligands and PET ligands, where available) within a family of targets and additional commentary highlights whether species differences or ligand metabolism are potential confounding factors.

We recommend that any citations to information in the Guide be presented in the following format:

ALEXANDER, S.P.H., MATHIE, A. \& PETERS, J.A. (2007). Guide to Receptors and Channels (GRAC), 2nd edition (2007 Revision). Br. J. Pharmacol., 150 (Suppl. 1), S1-S168.

Stephen P.H. Alexander School of Biomedical Sciences and Institute of Neuroscience,

University of Nottingham Medical School,

Nottingham NG7 2UH, UK.

steve.alexander@nottingham.ac.uk

Alistair Mathie

Division of Cell \& Molecular Biology,

Imperial College London,

Biophysics Section,

Blackett Laboratory,

South Kensington Campus,

London SW7 9AZ, UK.

a.mathie@imperial.ac.uk

John A. Peters Neurosciences Institute, Division of Pathology and Neuroscience,

Ninewells Hospital and Medical School,

The University of Dundee,

Dundee DD1 9SY, UK.

j.a.peters@dundee.ac.uk

\section{References:}

ALEXANDER, S.P.H., MATHIE, A. \& PETERS, J.A. (2004). Guide to Receptors and Channels, 1st edition. Br. J. Pharmacol., 141 (Suppl. 1), $\mathrm{S} 1-\mathrm{S} 126$.

VANHOUTTE, P.M., HUMPHREY, P.P.A. \& SPEDDING, M. (1996). X. International Union of Pharmacology recommendation for nomenclature of new receptor subtypes. Pharmacol. Rev., 48, 1-2.

The British Journal of Pharmacology would like to thank Pfizer Global Research and Development for their generous support with this publication.

H.P. Rang FRS

Editor-in-Chief

British Journal of Pharmacology 